\title{
PROMOÇÃO DO AUTOCUIDADO A MULHERES MASTECTOMIZADAS
}

\author{
Aline Priscila Rego de Carvalho ${ }^{1}$, Taciana Mirella Batista dos Santos ${ }^{2}$, Francisca Márcia Pereira Linhares ${ }^{3}$
}

RESUMO: Este estudo descritivo e exploratório teve como objetivo elaborar plano de cuidados de enfermagem à mulheres mastectomizadas na promoção do autocuidado, tendo por base a análise dos discursos daquelas que foram submetidas à cirurgia de mastectomia entre janeiro e agosto de 2009. A pesquisa foi fundamentada na Teoria de Orem, a qual norteia a assistência de enfermagem em qualquer estágio da vida do paciente, seja na manutenção do bem estar, recuperação da saúde, prevenção de doença ou reabilitação. Dos discursos emergiram as categorias temáticas Ações educativas centradas no autocuidado, Assistência centrada nas necessidades da mulher mastectomizada e na escuta ativa envolvendo a rede de apoio social, e Reuniões grupais: estratégia facilitadora ao enfrentamento pessoal. Essas permearam as orientações e a forma de fornecê-las para a promoção do autocuidado no pós-operatório. No estudo pudemos perceber a limitação do conhecimento das mulheres acerca dos cuidados a serem tomados no perioperatório de mastectomia.

PALAVRAS-CHAVE: Autocuidado; Enfermagem; Mastectomia; Câncer de mama.

\section{PROMOTION OF SELF-CARE FOR MASTECTOMIZED WOMEN}

\begin{abstract}
This descriptive, exploratory study aimed to devise a nursing care plan for women who had undergone mastectomy, in the promotion of self-care, based on analysis of the discourses of those who had received the surgery between January and August 2009. The research was grounded in Orem's Theory, which guides nursing care at any stage of the patient's life, whether in maintenance of well-being, recuperation of health, prevention of illness or rehabilitation. The following themes emerged from the discourses: Educative actions centered on self-care, Care centered on the needs of mastectomized women and in active listening involving the network of social support, and Group meetings: a facilitative strategy for personal coping. These permeate the guidance and the way of providing it for promoting self-care in the post-operative phase. In the study, the authors perceived the limitations on the women's knowledge about the care to be taken in the peri-operative period. KEYWORDS: Self-care; Nursing; Mastectomy; Breast cancer.
\end{abstract}

\section{PROMOCIÓN DEL AUTOCUIDADO A MUJERES MASTECTOMIZADAS}

RESUMEN: Este estudio descriptivo y exploratorio tuvo como objetivo elaborar un plan de cuidados de Enfermería para las mujeres mastectomizadas en la promoción del autocuidado, con base en el análisis de los discursos de aquellas que fueron sometidas a la cirugia de mastectomía entre enero y agosto de 2009. La investigación fue fundamentada en la Teoría de Orem, la cual dirige la asistencia de Enfermería en cualquier estagio de la vida del paciente, sea en la manutención del bienestar, recuperación de la salud, prevención de enfermedad o rehabilitación. De los discursos, surgieron las categorías temáticas Acciones educativas centradas en el autocuidado, Asistencia centrada en las necesidades de la mujer mastectomizada y en la escucha activa con la red de apoyo social, y Reuniones de grupo: estrategia facilitadora para afrontamiento personal. Esas permearon las orientaciones y la forma de fornecerlas para la promoción del autocuidado en el posoperatorio. En el estudio fue posible percibir la limitación del conocimiento de las mujeres acerca de los cuidados necesarios en el perioperatorio de mastectomía.

PALABRAS CLAVES: Autocuidado; Enfermería; Mastectomía; Cáncer de mama.

*Artigo baseado na Monografia do Curso de Residência em Enfermagem do Hospital das Clínicas de Pernambuco, Universidade Federal de Pernambuco - UFPE.

${ }^{1}$ Enfermeira do Instituto de Medicina Integral Professor Fernando Figueira. Especialista em Saúde da Mulher. ${ }^{2}$ Enfermeira da Prefeitura Municipal do Recife-PE. Especialista em Nefrologia e em Saúde do Trabalhador.

${ }^{3}$ Enfermeira. Doutora em Enfermagem. Professora do Departamento e do Programa de Pós-Graduação em Enfermagem da UFPE.

Autor correspondente:

Recebido: $19 / 12 / 2011$

Taciana Mirella Batista dos Santos

Aprovado: 18/05/2012

Prefeitura Municipal do Recife

R. Pe. Nestor de Alencar, 7342 - 54450-220 -Jaboatão dos Guararapes-PE-Brasil

E-mail: tacianamirella@hotmail.com 


\section{INTRODUÇÃO}

O câncer (CA) mamário é uma neoplasia maligna, complexa pelos diferentes graus de agressividade tumoral e pelo potencial metastático. Ocorre, principalmente, após os 40 anos de idade, embora nos últimos anos tenha havido um aumento da incidência em mulheres mais jovens. Cerca de 27 mulheres morrem por dia vítimas do $\mathrm{CA}$ de mama e esses números não param de crescer. $\mathrm{O}$ tratamento é dificultado pelo estágio avançado em que a doença é descoberta, o que compromete os resultados da terapêutica e diminui a sobrevida das pacientes ${ }^{(1)}$.

A mama é ícone da identidade feminina apresentando funções no desempenho do papel de mãe e na sexualidade. É visualizada como órgão indispensável ao corpo feminino, representando na esfera afetiva elementos ligados à projeção da imagem e à autoestima. Além disso, a alta frequência contribui para que o CA de mama seja, provavelmente, o mais temido no gênero ${ }^{(2)}$. $\mathrm{O}$ cuidado às mulheres mastectomizadas requer, além de cuidados próprios da cirurgia, apoio emocional, objetivando melhor compreensão, adaptação e aceitação da autoimagem ${ }^{(3)}$.

Ao assistir à mulher com alteração da autoimagem deve-se compreender que essa pode trazer traumas de ordem física, emocional e social que influenciam negativamente na evolução do tratamento e comprometem a dinâmica familiar ${ }^{(4)}$. A assistência para este grupo de mulheres requer um olhar multidisciplinar, levando em consideração a complexidade do contexto que a envolve. Por este motivo, a atenção à saúde da mulher mastectonizada deve objetivar, além de cuidados com o local da cirurgia, a assistência integral, levando em consideração as reais necessidades, valorizando sua independência e autonomia.

Assim, na busca de subsídios para melhorar a qualidade de vida das mulheres mastectomizads, se reconhece a necessidade de uma sustentação teórica para nortear a prática de Enfermagem. A Teoria do Autocuidado estabelece conceitos para a prática de Enfermagem que permeiam o desenvolvimento do indivíduo, seja ele de forma particular ou inserido em seu meio (família/comunidade), nas ações do autocuidado $^{(5)}$. O autocuidado é o conjunto de atividades que o indivíduo inicia e executa em seu próprio benefício, para manter, promover, recuperar e/ou conviver com os efeitos e limitações das alterações de saúde ${ }^{(6)}$. Esta Teoria concebe o homem como agente do cuidado, capaz de promover melhora na sua qualidade de vida. Com base nessa Teoria o enfermeiro precisa traduzir para o paciente, numa linguagem compreensível, os cuidados que a ele podem ser delegados. Cuidados simples, mas que além de preservar atividade corporal melhoram a autoestima ${ }^{(7)}$.

Nesta perspectiva, o presente estudo teve o objetivo de identificar quais cuidados e como estes devem ser orientados às mulheres mastectomizadas para promoção do autocuidado.

\section{MÉTODO}

Este estudo, descritivo exploratório com abordagem qualitativa, foi realizado no ambulatório de Ginecologia do Hospital das Clínicas da Universidade Federal de Pernambuco (HC/UFPE), localizado na Cidade de Recife-Pernambuco.

O universo empírico considerado para o processo de avaliação foi composto por mulheres submetidas à cirurgia de mastectomia entre janeiro e agosto de 2009. Para a amostra foi usado o critério de saturação teórica das informações qualitativas perfazendo um total de $11 \mathrm{mu}-$ lheres, cuja seleção foi do tipo intencional ou proposital.

A coleta de dados ocorreu entre os meses de maio e setembro de 2009. O instrumento utilizado foi uma entrevista com roteiro semiestruturado com as seguintes questões norteadoras: Quais os cuidados que você gostaria de ser orientada após a realização da mastectomia?Fale-me como você gostaria de receber estas orientações?

As falas das mulheres foram gravadas em aparelho de áudio tipo MP4 e analisadas em um único contexto, utilizando a técnica de análise de conteúdo na modalidade temática, proposta por $\operatorname{Bardin}^{(8)}$. A partir de palavras e frases significativas, foram abstraídos e codificados os núcleos de sentido; destas codificações emergiram as subcategorias e as categorias temáticas.

Os temas identificados foram discutidos correlacionando as necessidades do autocuidado das mulheres mastectomizadas com os princípios norteados da Teoria do Autocuidado. Todos os procedimentos relacionados à coleta e análise dos dados só foram iniciados após aprovação do projeto de pesquisa pelo Comitê de Ética em Pesquisa do Centro de Ciências da Saúde da UFPE, sob o número de protocolo 051/2009. Com o intuito de garantir o anonimato das mulheres foi estabelecido codinomes de flores.

\section{RESULTADOS}

Participaram do estudo 11 mulheres, com faixa etária entre 32 e 82 anos, e média de 53,3 anos. Quatro eram casadas, três divorciadas, três solteiras e uma vi- 
úva. Duas eram analfabetas, quatro possuíam o ensino fundamental incompleto, uma o ensino fundamental completo, duas o ensino médio incompleto, uma havia concluído o ensino médio e uma possuía o ensino superior incompleto.

Dez mulheres não possuíam vínculo empregatício; quanto à profissão/ocupação havia diversidade de tarefas, na qual duas declararam ser autônomas, uma do lar, uma doméstica, duas costureiras, uma aposentada, uma auxiliar de enfermagem, uma agricultora, uma merendeira e uma bordadeira. Seis possuíam uma renda mensal de um salário mínimo, três entre dois e três salários mínimos e duas, acima de três salários mínimos.

O CA de mama fora diagnosticado entre seis e 12 meses atrás para 8 mulheres, duas haviam descoberto entre um e dois meses e uma há mais de um ano. Seis mulheres afirmaram não possuir histórico de CA de mama na família e cinco citaram possuir antecedentes, sendo a mãe referida por três mulheres e a prima por duas entrevistadas.

Quanto à cirurgia, 10 mulheres haviam realizado há mais de 30 dias e somente uma entre 8 e 15 dias, sendo que o tempo de alta hospitalar variou entre menos de 7 dias, para uma mulher a mais de 30 dias para 10 mulheres. Apenas uma afirmou não ter recebido orientações no momento da alta, uma não se lembrava da categoria profissional da pessoa que a orientou; as demais receberam orientações de mais de um profissional, sendo que sete referiram ter sido orientadas pelo médico, duas pela enfermeira, duas pelo psicólogo e duas afirmaram ter recebido orientações da nutricionista.

Mais da metade (seis) das mulheres referiu participação em grupo de atividade/orientação, destacando a reunião em grupo do ambulatório de mastologia. Somente uma das participantes considerou questionável a presença/apoio da família no período perioperatório, enquanto as demais afirmaram ser importante.

Após a análise dos dados surgiram três categorias temáticas:

Tema 1: Ações Educativas centradas no autocuidado

Nas falas das participantes deste estudo percebemos a existência de dúvidas quanto às atividades que devem ser realizadas no cotidiano e às restrições nas realização de atividades diárias:

[...] todos os cuidados que deve ter né, com quanto tempo você pode fazer algum serviço de casa. (Camomila)
[...] tem que dizer, né o que a gente pode fazer em casa, ou não pode fazer [...]. (Tulipa)

[...] falar pra pessoa o que a pessoa deve fazer, como deve se comportar em casa. Sobre fazer os serviços de casa, o tratamento em casa. (Papoula)

Observamos que elas sentiam a necessidade de ouvir a verdade, mas com palavras de conforto, mostrando o caminho para que estas mulheres tenham condições próprias para o enfrentamento desta nova vida, como nos recortes dos discursos:

[...] falar tudo, que elas não desanimem, que elas tenham autoestima. (Violeta)

Falar como ela deve enfrentar a realidade da vida que chegou. Eu sei que é ruim, mas [...]. (Dália)

Neste contexto, os recortes das falas revelaram que as ações educativas devem estar direcionadas à realização dos procedimentos específicos necessários e restrições após o procedimento cirúrgico:

[...] é importante, muito importante orientar, né o que tem que fazer. (Magnólia)

Não fazer esforço com o braço, fazer a fisioterapia com cuidado [...]. (Orquídea)

É isso que tem que falar, né que a pessoa deve ter uma higiene. (Jasmim)

O pós-operatório da mastectomia envolve diversos fatores: o curativo, o cuidado com o dreno, atenção com o membro homolateral e a continuação do tratamento após a cirurgia, sendo esses apontados como itens a serem incluídos nas informações transmitidas:

Essas coisas toda têm que falar que a gente precisa de ter cuidado. Também explicar o porquê da fragilidade do braço, por que eu não posso tomar injeção, por que eu não posso medir a pressão, por que o mosquito não pode picar, por que eu não posso levar quentura?[...] (Orquídea)

Falar pra pessoa o tratamento em casa, sobre como eu podia me alimentar. Tinha que fazer os curativos, né. Como fazer, ter assim um cuidado. (Papoula) 
Deve orientar mais com relação ao dreno, como você deve manusear ele. É importante a orientação dos curativos, e também ser orientado com relação à dormência que você vai sentir. (Rosa)

Ainda nessa perspectiva das orientações, as falas retratam as dúvidas quanto os hábitos de higiene e alimentação:

Falar, principalmente, boa alimentação, o repouso, a higiene, deve falar do que pode fazer, ou não. (Violeta)

[...] sobre comida, sobre cuidado na família. (Girassol)

Percebemos que a mulher mastectomizada pensa que, por ter algumas restrições quanto à realização de atividades, tem também restrições no lazer, o que as fizeram sugerir que a temática fosse abordada no momento das orientações:

Sobre cuidado na família, a questão do divertimento, ajuda muito, viu? (Girassol)

[...] orientasse melhor sobre, prá você sair durante o dia. (Dália)

Ao considerar a terapêutica do CA de mama, notamos que as participantes do estudo relatam a necessidade de orientações quanto aos tratamentos complementares que poderão realizar:

[...] do tratamento, o que vai ter após a cirurgia, qual $e ́$ [...] (Margarida)

Falar da volta ao médico, ser acompanhada pelo médico, ser acompanhada e dizer porquê vai ser acompanhada [...] (Orquídea)

[...] em relação a medicamento. (Dália)

Tema 2 - Assistência centrada nas necessidades da mulher mastectomizada e na escuta ativa envolvendo a rede de apoio social

A rede de apoio social à mulher mastectomizada é fundamental na assistência a essas mulheres. No estudo foi evidenciado, nas falas, que os profissionais que prestam as orientações devem ter competência e habilidade para transmitir essas informações, estabelecendo vínculo com a paciente:
É muito importante a orientação de alguém que entenda do assunto e passe pra as pessoas que não tão sabendo. (Camomila)

É importante a gente ter mais um esclarecimento. Precisa, assim, de uma orientação mais profunda, precisa dar uma orientação mais clara. Por exemplo, da massagem linfática eu não sabia que precisava [...] (Orquídea)

Ainda nesse contexto, foi relatado que essas informações devem ser transmitidas não só para as mulheres, mas também aos companheiros, família ou outras pessoas que estão participando da história de vida dessa paciente:

[...] explicando prá ela [a mãe] o que ela pode fazer prá mim. No momento de alta do hospital que ela tava me orientando. (Dália)

[...] eu tenho muita vontade e deve chamar os esposos da gente e ter uma palestra com eles. E eles devem também receber a orientação. (Girassol)

\section{Tema 3 - Reuniões grupais: estratégia facilitadora ao enfrentamento pessoal}

As reuniões em grupos foram citadas nas falas como fator facilitador para enfrentar o desconhecido após a cirurgia, sendo que essas não se resumem apenas ao direcionamento dos participantes do grupo por um profissional, mas consiste na troca de experiências, onde os próprios sujeitos podem conduzir a discussão:

[...] estou dizendo que a pessoa deve orientar bem, em qualquer lugar, em qualquer momento a pessoa deve te orientar. Poderia juntar as pessoas que fizeram a cirurgia prá dar as orientações. (Camomila)

[...] poderia juntar a família, os amigos, prá quem entende. Isso é ótimo prá gente. (Dália)

\section{DISCUSSÃO}

Na perspectiva da Teoria do Autocuidado, quando os problemas são identificados eles determinam as ações e o tipo de intervenção de enfermagem apro$\operatorname{priada}^{(9)}$. As mulheres acometidas pelo CA de mama enfrentam diversas situações estressoras, as quais vão 
desde o diagnóstico e expectativas relacionadas ao tratamento cirúrgico, quimioterápico e radioterápico, até o período de reabilitação. A cirurgia em si pode causar medo nas mulheres, mas quando o tratamento tem continuidade com a quimioterapia ou a radioterapia, $o$ sofrimento permanece. Isso acontece, principalmente pelas mudanças na autoimagem ${ }^{(10)}$.

No pós-operatório imediato a mulher mastectomizada vivencia momentos de estresse pelo ato cirúrgico que altera a sua imagem corporal. As repercussões psicológicas após a mastectomia, além de afetarem o social da mulher, podem contribuir para o surgimento de sintomas físicos, podendo interferir no autocuidado, sendo indispensável que a paciente esteja ciente das possíveis complicações a fim de minimizá-las obtendo ajuda quando necessitar ${ }^{(10)}$.

Uma das mudanças do estilo de vida é a aparência pessoal, podendo interferir na autoestima e consequente recuperação da paciente. A aparência é fundamental na reintegração da paciente. Dentro dessa abordagem, particularmente o sutiã deve ter modelo específico para quem fez mastectomia e outro para quem realizou a reconstrução mamária, que deve usá-lo sem costura e preferir roupas de malha compressiva que forneça segurança e firmeza aos movimentos e melhore a imagem corporal.

No caso único de mastectomia o sutiã deve ser usado com prótese externa com peso aproximado ao da mama contralateral com a finalidade de prevenir outras complicações, como desvios da coluna. Orienta-se que as próteses externas não sejam lavadas em tanquinhos, máquinas, nem colocadas em secadoras ou passadas a ferro, a lavagem deve ser feita com sabão neutro e água fria, secagem na sombra e depois de seco a peça deve permanecer 30 minutos no congelador ${ }^{(7)}$.

Além dos cuidados com a aparência e vestuário para atender as necessidades de autoestima, as limitações das atividades domésticas representaram um dos principais questionamentos levantados pelas mulheres participantes do estudo. Considerando que a mulher com CA de mama tem seu cotidiano de vida alterado, principalmente pela consequência do tratamento, ela passa a viver em um ambiente de ansiedade em virtude do medo do seu prognóstico e dependência de outra pessoa.

As atividades diárias são preocupações comuns tanto no pré-operatório como no pós-operatório, visto que a mulher, na maioria das vezes, é acostumada a cuidar do lar e da família e o fato de ter que ser cuidada gera um sentimento de angústia, preocupação e ansiedade. A apreensão não se resume apenas no seu processo de adoecimento, mas em tudo que ela deixa de fazer devido a sua limitação física. Em consequência, seu adoecimento, muitas vezes gera uma mudança na rotina familiar ${ }^{(3)}$. As atividades domésticas são possíveis de serem realizadas, desde que sejam por etapas, e que isso traga segurança à mulher ${ }^{(7)}$.

A alta precoce pode ser recomendada quando há suporte domiciliar adequado, boas condições de saúde e fácil acesso à equipe. Contudo, as mulheres devem receber orientações para que sejam capazes de realizar autocuidado $^{(11-12)}$. Frequentemente, essas mulheres recebem alta ainda com o sistema de drenagem. Esse fato contribui para a diminuição do risco de infecção nosocomial e possibilita uma reintegração mais rápida da paciente ao seu convívio social. Em contrapartida, as pacientes passam a necessitar de orientações amplas sobre os cuidados com esse sistema no pós-operatório para que assumam a capacidade de se autocuidar com segurança ${ }^{(13)}$.

Após avaliação do potencial do paciente para o desenvolvimento do autocuidado, inicia-se a preparação da mesma, da família ou do responsável pelo autocuidado para se tornar independente da atuação do enfermeiro ${ }^{(9)}$. Sobre o sistema de drenagem aspirativo é importante que seja orientado a necessidade de atenção ao aspecto da drenagem; observar alterações no local do dreno (hiperemia, exsudato, dor); medir o conteúdo drenado no mínimo duas vezes ao dia, sempre lavando as mãos antes e após o procedimento; anotar a data, hora e quantidade da drenagem; manter vigilância para prevenir obstruções, quando se deve realizar a ordenha leve $\mathrm{e}^{(7)}$.

A equipe de enfermagem deve estar atenta e comunicar aos demais membros da equipe de saúde se houver aumento da quantidade ou da secreção sanguinolenta, drenagem ausente em 24 horas, se a bolsa coletora encher de ar, presença de sinais flogísticos ou saída do dreno. Enquanto estiver usando o dreno, a mulher deve preferir roupas folgadas para melhor acomodá-lo; durante o transporte e ao colocá-lo no chão o mesmo deverá estar envolvido em bolsa plástica. No momento da higienização é necessário lavar o local de incisão com movimentos leves e depois secar com pano limpo passado a ferro e separado exclusivamente para esse fim. A explicação desses procedimentos de forma detalhada parece fundamental para que as mulheres tenham estímulo para o autocuidado ${ }^{(7)}$.

Após a cirurgia de mastectomia a mulher deve também dispensar cuidados extras com o membro homolateral, e caso não sejam tomadas as devidos cuidados, poderão ocorrer complicações sérias ${ }^{(1)}$. Além 
de exercícios de reabilitação, a mulher precisa tomar alguns cuidados com o braço operado: evitar tirar cutícula, depilar a axila, ou ferir o membro; durante manuseio com materiais que possam trazer risco de cortes deve-se usar luvas; não permitir a aferição de sua pressão e injeções no membro operado; evitar dormir sobre o esse membro, para não comprometer a circulação; não carregar peso; usar luvas para o uso de produtos de limpeza e/ou outros produtos que possam irritar a pele; evitar calor (água, sol, fornos); não fazer esforços repetitivos e movimentos bruscos.

No entanto, a mulher deve ser encorajada a realizar medidas de higiene e autocuidado. Outro cuidado é a realização da drenagem linfática, que deve ser orientada à paciente logo após a cirurgia, para estimular a circulação linfática superficial e prevenir o linfedema ${ }^{(7)}$.

No estudo observamos que grande parte das mulheres sentia a necessidade de receber informações sobre a dieta. Deve-se ainda evitar gorduras, pois sua ingestão faz com que haja uma formação maior de hormônios femininos, que podem propiciar o aparecimento do câncer de mama ${ }^{(11)}$.

Mulheres com câncer de mama notam limitado apoio ao exercício físico, por parte dos médicos e do pessoal de enfermagem; alegando que esses profissionais de saúde não são bem informados no que diz respeito aos benefícios e exercícios adequados à mulher com câncer de mama ${ }^{(14)}$. Já as pacientes submetidas ao tratamento fisioterápico diminuem seu tempo de recuperação e retornam mais rapidamente as atividades diárias ${ }^{(15)}$.

A evolução do tratamento do CA de mama torna indispensável uma abordagem multidisciplinar, considerando, não só o quadro patológico, mas também a reabilitação física, psicológica e profissional, além de se preocupar com a qualidade de vida após o tratamento ${ }^{(15)}$. O fornecimento de meios para que a mulher tenha autonomia deve ser projetado aos seus familiares ${ }^{(11)}$.

A alta hospitalar gera para a família um sentimento ambíguo, ao mesmo tempo em que se sente feliz e aliviada por ver seu ente voltando para casa, sente medo e insegurança de cuidar do mesmo, muitas vezes pela mudança no estilo de vida que a própria doença impõe à família ${ }^{(15-16)}$.

A implementação do plano de alta é mais um meio de que o enfermeiro dispõe para demonstrar seus conhecimentos e definir o seu papel na equipe de saúde. É importante ressaltar que os folhetos explicativos não substituem a função ou o papel do profissional. Cartilhas e folhetos não excluem a interação com o paciente, devem ser considerado mais uma etapa importante da Sistematização da Assistência de Enfermagem que direciona o plano e a implementação das ações ${ }^{(16)}$.

De acordo com Lei do Exercício Profissional 7.498/86 e a Resolução COFEN 358/09, a SAE fundamenta exercício de enfermagem. E é uma ferramenta indispensável e obrigatória que direciona a prática do cuidado. Assim, além do direito e dever legal do enfermeiro de realizar a prescrição de enfermagem cabe a ele avaliar e monitorar suas intervenções ${ }^{(17-18)}$.

\section{CONSIDERAÇÕES FINAIS}

No estudo podemos perceber a limitação do conhecimento das mulheres. Nenhuma delas relatou que o dreno deve ser mantido abaixo do local de inserção, tão pouco sobre a importância de estar atenta ao surgimento de sinais flogísticos, quer do local de inserção do dreno ou da incisão cirúrgica. Muitas se mostravam ansiosas e afirmavam a falta de conhecimento a respeito da dieta, as atividades que poderiam e/ou deveriam realizar, algumas não relataram cuidados indispensáveis a serem tomados com o membro homolateral e outras até referiram informações incorretas.

$\mathrm{Na}$ atenção a mulher mastectomizada é necessário o envolvimento da rede de apoio social e da equipe multidisciplinar. Os diversos olhares para uma mesma problemática abordará esta mulher de forma integral, ajudando-a no enfrentamento desde o diagnóstico e até a pós alta.

O enfermeiro, através de seu papel como educador e atuando dentro da equipe multidisciplinar, deve engajar-se na atenção às mulheres e utilizar-se desse momento para reafirmar o seu papel, promovendo a assistência centrada no autocuidado de maneira interativa, participativa e de acordo com as necessidades identificadas e verbalizadas por elas.

\section{REFERENCIAS}

1. Instituto Nacional de Câncer (INCA) [Internet]. Rio de Janeiro. [acesso em 18 jan 2009]. Disponível: http:// www.inca.gov.br.

2. Maieski VM, Sarquis LMM. Mulheres com câncer de mama em quimioterapia e sua influência sobre o trabalho. [acesso em 01 Maio 2012]. Cogitare enferm. 2007;12(3). Disponível: http://ojs.c3sl.ufpr.br/ojs2/index. $\mathrm{php} /$ cogitare/article/view/10031/6890.

3. Kalinke L, Kochla K, LabronicI L, Lima T, Visentin A, Testoni R. Evolução das pacientes submetidas a cirurgia de mama em drenagem aspirativa. Cogitare

Cogitare Enferm. 2012 Jul/Set; 17(3):485-91 
enferm. 2011;16(4). [acesso em 28 abr 2012]. Disponível: http://ojs.c3sl.ufpr.br/ojs2/index.php/cogitare/article/ view/25440/17061.

4. Barreto RAS, Suzuki K, Lima MA, Moreira AA. As necessidades de informação de mulheres mastectomizadas subsidiando a assistência de enfermagem. Rev. Eletr. Enferm. 2008;10(1). [acesso em 21 jan 2009]. Disponível: http://www.fen.ufg.br/ revista/v10/n1/v10n1a10.htm.

5. Vall J, Lemos KIL, Janebro ASI. O processo de reabilitação de pessoas portadoras de lesão medular baseado nas teorias de enfermagem de Wanda Horta, Dorothea Orem e Callista Roy: um estudo teórico. Cogitare enferm. 2005;10(3):63-70.

6. Orem DE. Nursing: concepts of practice. $4^{\text {th }}$ ed. New York: McGraw-Hill. 1995.

7. Silva SED, Vasconcelos EV, Santana MEH, Rodrigues ILA, Leite TV, Santos LMS, Sousa RF, Conceição VRM, Oliveira JL, Meireles WDN. Representações sociais de mulheres mastectomizadas e suas implicações para o autocuidado. Rev. bras. enferm. 2010;63(5). [acesso em 23 mar 2012]. Disponível: http://www.redalyc.org/src/ inicio/ArtPdfRed.jsp?iCve=267019591006.

8. Bardin L. Análise de Conteúdo. $1^{\mathrm{a}}$ ed. Lisboa: Edições 70; 2011.

9. Sampaio FAA. Assistência de enfermagem a paciente com colostomia: aplicação da teoria de Orem. Acta paul. enferm. 2008;21(1):94-100.

10. Stumm E, Maçalai C, Leite M, Rosanelli C. Estressores vivenciados por mulheres mastectomizadas integrantes de um grupo de apoio. Cogitare enferm. 2010;15(3). [acesso em 28 abril 2012]. Disponível: http://ojs.c3sl.ufpr. br/ojs2/index.php/cogitare/article/view/18893/12202.

11. Melo EM. Avaliação de orientações sistematizadas de enfermagem no pós-operatório de mulheres submetidas à mastectomia [Tese]. Fortaleza (CE): Universidade Federal do Ceará; 2007.

12. Panobianco MS, Parra MV, Almeida AM, Prado MAS, Magalhães PAP. Estudo da adesão as estratégias de prevenção e controle de linfedema em mastectomizadas. Esc. Anna Nery. 2009;13(1):161-8.

13. Biffi RG, Mamede MV. Dinâmica familiar: percepção de famílias de sobreviventes de câncer de mama. Esc. Anna Nery. 2009;13(1):131-9.
14. Pacheco MN, Filho AD, Melo DAS. Fisioterapia para o tratamento do linfedema no pós-operatório de mastectomia: revisão de literatura Rev. Fac. Ciênc. Méd. Sorocaba. 2011;13(4):4-7.

15. Pompeo DA, Pinto MH, Cesarino CB, Araújo RRD, Poletti NAA. Atuação do enfermeiro na alta hospitalar: reflexões a partir dos relatos de pacientes. Acta paul. enferm. 2007;20(3):345-50.

16. Pereira APS, Tessarini MM, Pinto MH, Oliveira VDC. Alta hospitalar: visão de um grupo de enfermeiras. Rev. enferm. UERJ. 2007;15(1):40-5.

17. Brasil. Lei n. ${ }^{0} 7.498$, de 25 de Junho de 1986. Dispõe sobrea regulamentação do exercício da enfermagem e dá outras providências. [Internet] 15 out 2009 [acesso em 10 jan 2010]. Disponível: http://corensp.org. $\mathrm{br} / 072005 /$

18. Brasil. Conselho Federal de Enfermagem. Resolução COFEN n ${ }^{\circ} 358 / 2009$. Dispõe sobre a Sistematização da Assistência de Enfermagem e a implementação do Processo de Enfermagem em ambientes, públicos ou privados, em que ocorre o cuidado profissional de enfermagem, e dá outras providências. [Internet] 25 jun 2009 [acesso em 11 jan 2010]. Disponível: http:// site.portalcofen.gov.br/node/4384 